\section{Uso de diferentes critérios para avaliação da inadequação do pré-natal: um estudo de base populacional no extremo Sul do Brasil}

\author{
Use of different criteria to assess inadequate \\ prenatal care: a population-based study in \\ Southern Brazil
}

\author{
Uso de diferentes criterios para la evaluación de la \\ inadecuación del cuidado prenatal: un estudio de \\ base poblacional en el extremo Sur de Brasil
}




\section{Introdução}

O pré-natal compreende um dos mais completos conjuntos de cuidados em saúde oferecido a um grupo populacional específico. Seu potencial de impacto sobre a morbimortalidade materno-infantil é amplamente conhecido, tanto pela redução na ocorrência de trabalho de parto prematuro, doença hipertensiva específica da gestação e diabetes gestacional quanto pela diminuição da severidade dos casos 1,2. Estimase que um quarto dos óbitos infantis e todos os óbitos maternos decorram da ausência da oferta de pré-natal adequado ${ }^{3}$. Por essa razão, oferecer esse tipo de serviço deveria ser prioridade absoluta em todas as esferas de governo (municipal, estadual ou federal) 2,3.

Vários índices buscando avaliar a qualidade do pré-natal são conhecidos. Um dos primeiros índices foi proposto por Kessner em 1973, posteriormente modificado por Kotelchuck em 1994 4. No Brasil, uma das primeiras propostas de avaliação da adequação do pré-natal, também com base no índice proposto por Kessner, foi feita por Takeda em 1993 5. Todos esses índices baseavam-se no número de consultas realizadas e na idade gestacional de início do pré-natal. Em 2000, ao instituir o Programa de Humanização do Pré-Natal (PHPN) 6, o Ministério da Saúde definiu um conjunto de procedimentos mínimos que todas as gestantes deveriam receber durante o exame que, além do número de consultas e da idade gestacional, levava em conta a realização de alguns exames clínicos e laboratoriais. Em 2001, Silveira et al. 7 acrescentaram ao pré-natal adequado a realização de hemograma, exame de urina e sorológico para sífilis. Em 2003, Coimbra et al. 8 criaram, baseando-se em recomendações do Ministério da Saúde, um índice que, além do número de consultas e mês de início do prénatal, incluía o número de consultas ajustadas à idade gestacional do parto.

No Brasil, tanto o acesso a serviços de saúde quanto à qualidade do que é oferecido varia marcadamente entre regiões, entre municípios e, às vezes, entre usuários 1,2,9. Além disso, é sabido que, via de regra, quanto maior a necessidade em saúde, menor a oferta e, não raramente, pior a qualidade dos serviços oferecidos. Isso contribui, no caso do pré-natal, para o aumento da sua inadequação 1 .

O presente estudo mediu a prevalência de inadequação do pré-natal entre puérperas residentes no Município de Rio Grande, Rio Grande do Sul, Brasil, que tiveram filhos em 2010, baseada nos critérios utilizados por Takeda 5 , Coimbra et al. 8 e Silveira et al. 7 . Além disso, identificou fatores associados à realização de pré-natal inadequado para cada um desses critérios.

\section{Material e métodos}

Este trabalho foi realizado em Rio Grande, município localizado na região litorânea na chamada Metade Sul do Rio Grande do Sul, que possui cerca de 200 mil habitantes. Em 2010 ocupava, dentre os 496 municípios do estado, a quarta posição em termos de Produto Interno Bruto (PIB) e apresentava Índice de Desenvolvimento Humano (IDH) de 0,744 10.

Quando este estudo foi conduzido, Rio Grande possuía 32 unidades básicas de saúde (UBS), cinco ambulatórios e dois hospitais gerais: Santa Casa de Misericórdia e o Hospital Universitário da Universidade Federal do Rio Grande. Praticamente todos os nascimentos do município $(99,5 \%)$ ocorriam nas maternidades desses hospitais.

Para ser incluída no estudo, a puérpera deveria residir no Município de Rio Grande, ter tido filho de 1o de janeiro a 31 de dezembro de 2010 e o recém-nascido ter alcançado peso igual ou superior a $500 \mathrm{~g}$ ou pelo menos 20 semanas de idade gestacional.

O delineamento utilizado neste trabalho foi do tipo transversal (ou de prevalência). O cálculo do tamanho amostral foi realizado com o software Epi Info 6.04 (Centers for Disease Control and Prevention, Atlanta, Estados Unidos). Considerou-se a prevalência de inadequação segundo Takeda, Coimbra et al. e Silveira et al. de 30\%, $25 \%$ e $60 \%$, respectivamente, margem de erro de dois pontos percentuais e nível de $95 \%$ de confiança. Utilizando-se esses parâmetros, o estudo deveria incluir pelo menos 2.309 puérperas. Para exposições variando de $17 \%$ a $76 \%$, frequência de realização de pré-natal inadequado no grupo não exposto de pelo menos $12 \%$ para Takeda e Coimbra et al. e de $28 \%$ para Silveira et al., razão de prevalências de 1,4, acrescidas de $10 \%$ para eventuais perdas e $15 \%$ para controle de potenciais fatores de confusão, o presente estudo deveria incluir pelo menos 2.304 recém-nascidos.

Uma equipe de entrevistadoras graduadas em serviço social foi capacitada para coleta de dados. O estudo piloto foi realizado nessas duas maternidades na primeira quinzena de dezembro de 2009 e teve por objetivo testar o questionário a ser utilizado e avaliar a logística do trabalho.

A coleta de dados foi realizada somente após a apresentação e assinatura do Termo de Consentimento Livre e Esclarecido (TCLE). Os dados foram obtidos por meio de questionário único 
pré-codificado com quase a totalidade das questões fechadas, aplicado às mães em até 24 horas após o parto. Nesse inquérito foram investigadas as características demográficas (idade, cor da pele autorreferida e se vivia com companheiro), socioeconômicas (renda familiar mensal em salários mínimos, escolaridade em anos completos com aprovação e se realizou trabalho remunerado no período gestacional), habitacionais (número de moradores por domicílio e índice de bens), reprodutivas maternas (paridade, ocorrência prévia de aborto e planejamento da gravidez atual), utilização de serviços de saúde (número de consultas realizadas e período de início do pré-natal, local de realização da maioria destas consultas e frequência de realização de exame de sangue, de urina e sorologia para sífilis a que foi submetida durante a gestação) e presença de morbidade materna autorreferida (hipertensão arterial sistêmica, diabetes mellitus, depressão, anemia, ameaça de aborto, trabalho de parto prematuro, corrimento vaginal, infecção do trato urinário e sangramento vaginal).

Os desfechos deste estudo foram constituídos pela inadequação do pré-natal segundo critérios utilizados por Takeda, Coimbra et al. e Silveira et al. No caso de Takeda, considerou-se como tendo realizado pré-natal inadequado a gestante que compareceu a cinco consultas ou menos e/ ou que as tenha iniciado a partir do quinto mês de gestação. Para Coimbra et al., considerou-se como inadequado quando o pré-natal iniciou a partir do quinto mês de gestação e/ou tenha realizado cinco consultas ou menos para uma gestação a termo, ou menor número que o esperado para a idade gestacional no parto. A realização de quatro consultas ou menos se o parto ocorreu com idade gestacional entre 33 e 36 semanas, se entre 29 e 32 semanas, três ou menos consultas, e se a duração da gravidez foi menor que 24 semanas a realização de uma consulta foi considerada como inadequado. No caso de Silveira et al., foi considerado como inadequado a gestante que iniciou o pré-natal a partir do quinto mês e/ou realizou cinco consultas ou menos e/ou realizou somente um ou nenhum dos seguintes exames laboratoriais: hemograma, exame qualitativo de urina e exame sorológico para sífilis (VDRL - Venereal Disease Research Laboratory).

Os questionários foram duplamente digitados utilizando-se o software EpiData 3.1 (Epidata Assoc., Odense, Dinamarca). Posteriormente, as digitações foram comparadas e corrigidas por meio do software Epi Info 6.04. Após, os dados foram analisados no pacote estatístico Stata 11.2 (Stata Corp., College Station, Estados Unidos), em que foi realizada a análise bivariada buscando medir o nível de associação entre as variáveis independentes e cada um dos três desfechos. Para a comparação entre as proporções foi utilizado o teste do qui-quadrado. Em seguida, foram realizadas as análises brutas e ajustadas mediante regressão de Poisson com variância robusta 11, obedecendo ao modelo hierárquico previamente definido. Nesse modelo, as variáveis de um determinado nível são ajustadas para aquelas do mesmo nível e de níveis anteriores. Por exemplo, a variável índice de bens presente no segundo nível, foi ajustada para aglomeração familiar situada neste mesmo nível e para todas aquelas do nível anterior (nível I: idade da mãe, cor da pele, viver com companheiro, escolaridade da mãe, renda familiar e realização de trabalho remunerado durante a gravidez). Assim, cada bloco de variáveis de um determinado nível foi incluído na análise e mantiveram-se no modelo todas aquelas variáveis cujo valor de $\mathrm{p}$ foi $\leq$ 0,20 em relação a cada um dos desfechos. No primeiro nível ficaram as variáveis demográficas e socioeconômicas; no segundo, as variáveis habitacionais; no terceiro, foram colocadas as variáveis reprodutivas maternas, e no quarto nível foram incluídas as variáveis relativas à utilização de serviços de saúde e padrão de morbidade da mãe.

Esse modelo de análise obedeceu ao proposto por Victora et al. 12. A significância estatística de cada variável no modelo foi avaliada usandose o teste de heterogeneidade e de tendência de Wald de acordo com o seu tipo 13 .

O projeto de pesquisa foi submetido e aprovado pelo Comitê de Ética em Pesquisa na Área da Saúde (CEPAS) da Universidade Federal do Rio Grande (processo 117/2009) e da Santa Casa de Misericórdia de Rio Grande (processo 009/2009).

\section{Resultados}

Foram entrevistadas 2.395 puérperas $(97,2 \%$ do total de nascimentos). Todas residiam no $\mathrm{Mu}-$ nicípio de Rio Grande e tiveram filho em 2010. Dentre as 69 restantes, 51 não foram encontradas e 18 recusaram-se a participar do estudo. Entre as participantes, um quinto delas era adolescente (<20 anos), $70 \%$ eram de cor da pele branca, $92 \%$ possuíam cinco anos ou mais de escolaridade, $50 \%$ tinham renda familiar $\leq 2$ (salários mínimos) e $43 \%$ eram primíparas.

Cerca de $5 \%$ das entrevistadas não realizaram uma única consulta de pré-natal. A prevalência de realização de pré-natal inadequado segundo critério utilizado por Takeda foi de $28,4 \%$ (IC95\%: 26,6-30,2); segundo Coimbra et al., de 26,8\% (IC95\%: 25,0-28,5); e de acordo com Silveira et al., de 58,2\% (IC95\%: 56,1-60,1). 
Na Tabela 1, observa-se que a prevalência de inadequação do pré-natal segundo Takeda variou de $6,8 \%$ entre aquelas com 12 anos ou mais de escolaridade a $47,7 \%$ entre gestantes pertencentes ao primeiro quartil de bens. Ainda segundo esse critério, quase todas as variáveis, exceto história prévia de aborto e intercorrências na gestação, mostraram-se significativamente associadas à inadequação após análise ajustada.

A Tabela 2 mostra também ampla variação na prevalência de inadequação do pré-natal segundo critério utilizado por Coimbra et al., variando de 6,8\% para aquelas com 12 anos ou mais de escolaridade a $45,1 \%$ para mães com três ou mais filhos. As únicas variáveis não associadas à inadequação após análise ajustada foram: realização de atividade remunerada no período gestacional e história prévia de aborto.

A Tabela 3 apresenta resultados da inadequação do pré-natal conforme critério utilizado por Silveira et al. Segundo esse critério, a prevalência de inadequação variou de $46 \%$ entre gestantes com renda familiar $\geq 4$ salários mínimos a $72,8 \%$ para as pertencentes ao pior quartil do índice de bens. A análise ajustada mostrou que viver sem companheiro, possuir

Tabela 1

Análises bruta e ajustada para fatores associados à inadequação do pré-natal segundo critério proposto por Takeda 5 * Rio Grande, Rio Grande do Sul, Brasil, 2010 ( $n=2.395$ ).

\begin{tabular}{|c|c|c|c|}
\hline \multirow[t]{2}{*}{ Nível/Variável } & \multirow{2}{*}{$\begin{array}{c}\text { Prevalência de } \\
\text { inadequação (\%) }\end{array}$} & \multicolumn{2}{|c|}{ RP (IC95\%) } \\
\hline & & Bruta & Ajustada \\
\hline \multicolumn{4}{|l|}{ I } \\
\hline Idade da mãe (anos completos) & & $\mathrm{p}<0,001$ ** & $p=0,009 \star \star$ \\
\hline $11-19$ & 37,5 & 1,00 & 1,00 \\
\hline $20-24$ & 33 & $0,88(0,75-1,03)$ & $1,21(1,02-1,42)$ \\
\hline $25-29$ & 22,3 & $0,59(0,49-0,72)$ & $0,92(0,76-1,11)$ \\
\hline 30 ou mais & 23,8 & $0,63(0,53-0,76)$ & $1,00(0,83-1,20)$ \\
\hline Cor da pele & & $p<0,001 * \star \star$ & $p<0,001 * \star \star$ \\
\hline Branca & 24,7 & 1,00 & 1,00 \\
\hline Parda & 33,2 & $1,34(1,15-1,56)$ & $1,13(0,98-1,30)$ \\
\hline Preta & 44,5 & $1,80(1,53-2,12)$ & $1,45(1,23-1,70)$ \\
\hline Vive com o companheiro & & $p<0,001 * \star \star$ & $\mathrm{p}<0,001 * \star \star$ \\
\hline Não & 47,6 & $1,94(1,71-2,21)$ & $1,63(1,43-1,87)$ \\
\hline Sim & 27,5 & 1,00 & 1,00 \\
\hline Escolaridade (anos completos) & & $\mathrm{p}<0,001$ ** & $p<0,001 * \star$ \\
\hline $0-4$ & 46,1 & $6,72(4,14-10,90)$ & $3,80(2,29-6,31)$ \\
\hline $5-8$ & 40,3 & $5,88(3,69-9,37)$ & $3,32(2,04-5,42)$ \\
\hline $9-11$ & 20,4 & $2,97(1,85-4,77)$ & $2,05(1,27-3,32)$ \\
\hline 12 ou mais & 6,8 & 1,00 & 1,00 \\
\hline Renda familiar (salários mínimos) & & $\mathrm{p}<0,001 \star \star$ & $p<0,001 * \star$ \\
\hline $0,0-0,9$ & 40,5 & $3,49(2,62-4,65)$ & $1,89(1,41-2,54)$ \\
\hline $1,0-1,9$ & 36,8 & $3,17(2,40-4,18)$ & $1,86(1,39-2,48)$ \\
\hline $2,0-3,9$ & 22,2 & $1,91(1,42-2,56)$ & $1,41(1,05-1,88)$ \\
\hline 4,0 ou mais & 11,6 & 1,00 & 1,00 \\
\hline $\begin{array}{l}\text { Se a mãe exerceu atividade } \\
\text { remunerada durante a gravidez }\end{array}$ & & $\mathrm{p}<0,001 * \star \star$ & $p=0,036 * \star \star$ \\
\hline Não & 34,2 & $1,94(1,71-2,21)$ & $1,17(1,01-1,35)$ \\
\hline Sim & 20,7 & 1,00 & 1,00 \\
\hline
\end{tabular}

(continua) 
Tabela 1 (continuação)

\begin{tabular}{|c|c|c|c|}
\hline \multirow{2}{*}{ Nível/Variável } & \multirow{2}{*}{$\begin{array}{l}\text { Prevalência de } \\
\text { inadequação (\%) }\end{array}$} & \multicolumn{2}{|c|}{ RP (IC95\%) } \\
\hline & & Bruta & Ajustada \\
\hline \multicolumn{4}{|l|}{ II } \\
\hline Número de moradores por domicílio & & $p<0,001$ ** & $p<0,001$ ** \\
\hline $1-2$ & 17,1 & 1,00 & 1,00 \\
\hline $3-4$ & 29 & $1,69(1,42-2,02)$ & $1,69(1,43-2,00)$ \\
\hline 5 ou mais & 45,9 & $2,68(2,24-3,20)$ & $2,24(1,86-2,69)$ \\
\hline Índice de bens em quartis & & $p<0,001 * \star$ & $p<0,001 * \star$ \\
\hline Primeiro (pior) & 47,7 & $3,43(2,77-4,26)$ & $2,03(1,59-2,58)$ \\
\hline Segundo & 30,7 & $2,21(1,75-2,79)$ & $1,48(1,16-1,88)$ \\
\hline Terceiro & 21,4 & $1,54(1,20-1,99)$ & $1,32(1,03-1,69)$ \\
\hline Quarto (melhor) & 13,9 & 1,00 & 1,00 \\
\hline \multicolumn{4}{|l|}{ III } \\
\hline Número de filhos tidos & & $p<0,001 * *$ & $p=0,006$ ** \\
\hline Nenhum & 19 & 1,00 & 1,00 \\
\hline $1-2$ & 29,4 & $1,55(1,32-1,82)$ & $1,66(0,64-4,32)$ \\
\hline 3 ou mais & 47,2 & $2,49(2,13-2,91)$ & $2,11(0,81-5,50)$ \\
\hline Se teve algum aborto & & $p<0,001$ *** & $p=0,091 * * *$ \\
\hline Não & 37,9 & 1,00 & 1,00 \\
\hline Sim & 26,3 & $0,69(0,57-0,84)$ & $0,85(0,70-1,03)$ \\
\hline Se planejou a gravidez & & 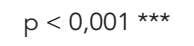 & $p<0,001 * \star \star$ \\
\hline Sim & 15,1 & 1,00 & 1,00 \\
\hline Mais ou menos & 24,5 & $1,62(1,22-2,17)$ & $1,57(1,14-2,16)$ \\
\hline Não & 37,7 & $2,50(2,11-2,98)$ & $1,77(1,43-2,19)$ \\
\hline \multicolumn{4}{|l|}{ IV } \\
\hline Tipo de médico que fez pré-natal & & $p<0,001 * \star \star$ & $p<0,001 * \star \star$ \\
\hline Convênio/Particular & 12,4 & 1,00 & 1,00 \\
\hline ESF & 23,9 & $1,92(1,52-2,43)$ & $1,01(0,74-1,37)$ \\
\hline Tradicional & 41,3 & $3,31(2,68-4,10)$ & $1,51(1,14-2,01)$ \\
\hline Ambulatório & 38,8 & $3,11(2,56-3,79)$ & $1,69(1,29-2,21)$ \\
\hline Intercorrências durante a gestação \# & & $p<0,001$ ** & $p=0,129 * *$ \\
\hline Nenhuma & 35,9 & $1,52(1,24-1,85)$ & $1,26(0,99-1,59)$ \\
\hline $1-2$ & 26,9 & $1,14(0,94-1,37)$ & $1,10(0,89-1,37)$ \\
\hline 3 ou mais & 23,6 & 1,00 & 1,00 \\
\hline Total & 28,4 & & \\
\hline
\end{tabular}

ESF: Estratégia Saúde da Família; IC95\%: intervalo de 95\% de confiança; RP: razão de prevalência.

* Realização de menos de seis consultas de pré-natal com início a partir do quinto mês de gestação;

** Teste de tendência linear;

*** Teste de heterogeneidade;

\# Inclui o relato das seguintes doenças: hipertensão arterial sistêmica, diabetes mellitus, depressão, anemia, ameaça de aborto, trabalho de parto prematuro, corrimento vaginal, infecção do trato urinário e sangramento vaginal nos últimos três meses de gestação.

baixa escolaridade e/ou renda familiar, conviver com maior número de pessoas no domicílio, ter baixa disponibilidade de bens no domicílio, maior paridade, realizar pré-natal com médi- co em UBS tradicional ou ambulatório e não apresentar intercorrências na gestação conferiram maior probabilidade de realização de pré-natal inadequado. 
Tabela 2

Análises bruta e ajustada para fatores associados à inadequação do pré-natal segundo critério proposto por Coimbra et al. 8 *. Rio Grande, Rio Grande do Sul, Brasil, 2010 ( $n=2.395)$.

\begin{tabular}{|c|c|c|c|}
\hline \multirow[t]{2}{*}{ Nível/Variável } & \multirow{2}{*}{$\begin{array}{c}\text { Prevalência de } \\
\text { inadequação (\%) }\end{array}$} & \multicolumn{2}{|c|}{ RP (IC95\%) } \\
\hline & & Bruta & Ajustada \\
\hline \multicolumn{4}{|l|}{ I } \\
\hline Idade da mãe (anos completos) & & $p<0,001 * \star$ & $p=0,004 * \star$ \\
\hline $11-19$ & 33,7 & 1,00 & 1,00 \\
\hline $20-24$ & 31,8 & $0,94(0,79-1,12)$ & $1,29(1,08-1,53)$ \\
\hline $25-29$ & 21,8 & $0,65(0,53-0,79)$ & $0,99(0,81-1,21)$ \\
\hline 30 ou mais & 22,1 & $0,65(0,54-0,79)$ & $1,02(0,83-1,24)$ \\
\hline Cor da pele & & $p<0,001 * \star *$ & $p<0,001 * * *$ \\
\hline Branca & 23,1 & 1,00 & 1,00 \\
\hline Parda & 31,8 & $1,37(1,17-1,60)$ & $1,15(0,99-1,34)$ \\
\hline Preta & 41,6 & $1,80(1,51-2,14)$ & $1,44(1,21-1,72)$ \\
\hline Vive com o companheiro & & $\mathrm{p}<0,001 * \star \star$ & $p=0,001 \star \star \star$ \\
\hline Não & 44,9 & $1,94(1,70-2,22)$ & $1,65(1,43-1,89)$ \\
\hline Sim & 23,1 & 1,00 & 1,00 \\
\hline Escolaridade (anos completos) & & $p<0,001 * \star$ & $p<0,001 * \star$ \\
\hline $0-4$ & 44,5 & $6,49(3,99-10,55)$ & $3,74(2,24-6,23)$ \\
\hline $5-8$ & 37,5 & $5,47(3,43-8,73)$ & $3,15(1,92-5,15)$ \\
\hline $9-11$ & 19,2 & $2,80(1,74-4,51)$ & $1,94(1,20-3,15)$ \\
\hline 12 ou mais & 6,8 & 1,00 & 1,00 \\
\hline Renda familiar (salários mínimos) & & $p<0,001 * \star$ & $p<0,001 * *$ \\
\hline $0,0-0,9$ & 38,4 & $3,45(2,56-4,63)$ & $1,89(1,39-2,57)$ \\
\hline $1,0-1,9$ & 34,5 & $3,10(2,33-4,13)$ & $1,85(1,37-2,50)$ \\
\hline $2,0-3,9$ & 20,7 & $1,86(1,37-2,52)$ & $1,38(1,02-1,87)$ \\
\hline 4,0 ou mais & 11,1 & 1,00 & 1,00 \\
\hline $\begin{array}{l}\text { Se a mãe exerceu atividade } \\
\text { remunerada durante a gravidez }\end{array}$ & & $p<0,001 * \star \star$ & $p=0,098 * \star \star$ \\
\hline Não & 31,9 & $1,60(1,38-1,85)$ & $1,13(0,98-1,32)$ \\
\hline $\operatorname{Sim}$ & 19,9 & 1,00 & 1,00 \\
\hline \multicolumn{4}{|l|}{ II } \\
\hline Número de moradores por domicílio & & $p<0,001 * \star$ & $p<0,001 * \star$ \\
\hline $1-2$ & 16,2 & 1,00 & 1,00 \\
\hline $3-4$ & 27,7 & $1,71(1,42-2,05)$ & $1,70(1,42-2,03)$ \\
\hline 5 ou mais & 42,5 & $2,62(2,18-3,16)$ & $2,18(1,80-2,65)$ \\
\hline Índice de bens (quartis) & & $p<0,001 * \star$ & $\mathrm{p}<0,001 * \star$ \\
\hline Primeiro (pior) & 44,5 & $3,33(2,66-4,16)$ & $1,92(1,49-2,48)$ \\
\hline Segundo & 28,5 & $2,13(1,68-2,71)$ & $1,41(1,09-1,81)$ \\
\hline Terceiro & 20,1 & $1,54(1,19-1,99)$ & $1,31(1,01-1,69)$ \\
\hline Quarto (melhor) & 13,4 & 1,00 & 1,00 \\
\hline \multicolumn{4}{|l|}{ III } \\
\hline Número de filhos tidos & & $p<0,001 * \star$ & $p=0,005 * \star$ \\
\hline Nenhum & 17,3 & 1,00 & 1,00 \\
\hline $1-2$ & 27,9 & $1,61(1,36-1,91)$ & $1,58(0,61-4,11)$ \\
\hline 3 ou mais & 45,1 & $2,60(2,21-3,07)$ & $2,04(0,78-5,31)$ \\
\hline
\end{tabular}

(continua) 
Tabela 2 (continuação)

\begin{tabular}{|c|c|c|c|}
\hline \multirow[t]{2}{*}{ Nível/Variável } & \multirow{2}{*}{$\begin{array}{c}\text { Prevalência de } \\
\text { inadequação (\%) }\end{array}$} & \multicolumn{2}{|c|}{ RP (IC95\%) } \\
\hline & & Bruta & Ajustada \\
\hline \multicolumn{4}{|l|}{ III } \\
\hline Se teve algum aborto & & $p<0,001 \star \star \star$ & $p=0,083 * \star \star$ \\
\hline Não & 36,2 & 1,00 & 1,00 \\
\hline Sim & 24,8 & $0,68(0,56-0,84)$ & $0,84(0,69-1,02)$ \\
\hline Se planejou a gravidez & & $p<0,001 * \star \star$ & $p<0,001 * \star \star$ \\
\hline $\operatorname{Sim}$ & 13,1 & 1,00 & 1,00 \\
\hline Mais ou menos & 23,0 & $1,76(1,30-2,38)$ & $1,71(1,21-2,42)$ \\
\hline Não & 36,2 & $2,77(2,30-3,33)$ & $2,00(1,58-2,53)$ \\
\hline \multicolumn{4}{|l|}{ IV } \\
\hline Tipo de médico que fez pré-natal & & $p<0,001 * \star \star$ & $p<0,001 * \star \star$ \\
\hline Convênio/Particular & 11,8 & 1,00 & 1,00 \\
\hline ESF & 21,0 & $1,78(1,39-2,28)$ & $0,95(0,69-1,31)$ \\
\hline Tradicional & 38,1 & $3,22(2,58-4,02)$ & $1,47(1,10-1,98)$ \\
\hline Ambulatório & 36,8 & $3,11(2,54-3,82)$ & $1,74(1,32-2,30)$ \\
\hline Intercorrências durante a gestação \# & & $p<0,001 * *$ & $\mathrm{p}<0,028$ ** \\
\hline Nenhuma & 35,5 & $1,73(1,40-2,14)$ & $1,37(1,06-1,76)$ \\
\hline $1-2$ & 25,1 & $1,22(1,00-1,50)$ & $1,14(0,90-1,44)$ \\
\hline 3 ou mais & 20,5 & 1,00 & 1,00 \\
\hline Total & 26,8 & & \\
\hline
\end{tabular}

ESF: Estratégia Saúde da Família; IC95\%: intervalo de 95\% de confiança; RP: razão de prevalência.

* Pré-natal inadequado por Coimbra et al.: se iniciou a partir do quinto mês de gestação e/ou quando realizadas cinco ou menos consultas para uma gestação a termo; realização de quatro consultas ou menos se o parto ocorreu com idade gestacional entre 33 e 36 semanas; se entre 29 e 32 semanas, três ou menos consultas; realização de duas consultas ao menos se o parto ocorreu entre 24 e 28 semanas; e se realizada somente uma consulta quando o parto ocorreu com 23 semanas ou menos de gestação;

** Teste de tendência linear;

*** Teste de heterogeneidade;

\# Doença hipertensiva específica da gestação, hipertensão arterial sistêmica, diabetes mellitus, depressão, anemia, ameaça de aborto, trabalho de parto prematuro, corrimento vaginal, infecção do trato urinário, sangramento vaginal nos últimos três meses de gestação.

\section{Discussão}

Este trabalho mostrou que, no Município de Rio Grande, pelo menos um quarto das gestantes realizou pré-natal inadequado. Mostrou ainda que há enorme iniquidade no acesso a esse tipo de serviço e, como era de se esperar, quanto mais exigente o critério utilizado maior a prevalência de inadequação.

Ao interpretar esses resultados é preciso considerar que os dados aqui apresentados foram obtidos, como na maioria dos estudos que tratam deste assunto, com base nas informações prestadas pela mãe, mas não checadas na carteira da gestante. Além disso, apesar do controle para fatores confundidores, o delineamento utilizado foi do tipo transversal, o que dificulta o estabelecimento de relação de causalidade. Por fim, em virtude de o desfecho obrigatoriamente ser dicotômico em função do tipo de análise realizada (regressão logística), a categoria inadequada incluiu também as mães cujo pré-natal foi classificado como intermediário nos três critérios referidos.

A revisão da literatura mostrou que, no Brasil, é baixa a utilização de critérios visando a avaliar a qualidade da assistência pré-natal. Em geral, as avaliações que tratam desse assunto são limitadas à contagem do número de consultas realizadas, com especial interesse na proporção daquelas que completam pelo menos seis consultas, número mínimo recomendado pelo Ministério da Saúde 6 
Tabela 3

Análises bruta e ajustada para fatores associados à inadequação do pré-natal segundo critério proposto por Silveira et al. 7 * . Rio Grande, Rio Grande do Sul, Brasil, 2010 ( $n=2.395$ ).

\begin{tabular}{|c|c|c|c|}
\hline \multirow[t]{2}{*}{ Nível/Variável } & \multirow{2}{*}{$\begin{array}{c}\text { Prevalência de } \\
\text { inadequação (\%) }\end{array}$} & \multicolumn{2}{|c|}{ RP (IC95\%) } \\
\hline & & Bruta & Ajustada \\
\hline \multicolumn{4}{|l|}{1} \\
\hline Idade da mãe (anos completos) & & $p<0,001$ ** & $p=0,338 * \star$ \\
\hline $11-19$ & 67,4 & 1,00 & 1,00 \\
\hline $20-24$ & 58,7 & $0,87(0,79-0,95)$ & $0,99(0,90-1,08)$ \\
\hline $25-29$ & 57,3 & $0,85(0,77-0,93)$ & $1,02(0,92-1,12)$ \\
\hline 30 ou mais & 52,4 & $0,78(0,70-0,85)$ & $0,93(0,84-1,03)$ \\
\hline Cor da pele & & $p<0,001 * \star \star$ & $p=0,071 * \star \star$ \\
\hline Branca & 55,7 & 1,00 & 1,00 \\
\hline Parda & 61,7 & $1,11(1,02-1,20)$ & $1,04(0,96-1,12)$ \\
\hline Preta & 67,6 & $1,21(1,10-1,34)$ & $1,12(1,01-1,23)$ \\
\hline Vive com o companheiro & & $\mathrm{p}<0,001 * \star \star$ & $\mathrm{p}<0,001 * * *$ \\
\hline Não & 72,2 & $1,30(1,21-140)$ & $1,20(1,12-1,30)$ \\
\hline Sim & 55,3 & 1,00 & 1,00 \\
\hline Escolaridade (anos completos) & & $p<0,001$ ** & $p<0,001$ ** \\
\hline $0-4$ & 71,7 & $1,91(1,59-2,30)$ & $1,63(1,34-1,99)$ \\
\hline $5-8$ & 68,2 & $1,82(1,54-2,15)$ & $1,55(1,29-1,86)$ \\
\hline $9-11$ & 52,2 & $1,39(1,17-1,65)$ & $1,27(1,06-1,52)$ \\
\hline 12 ou mais & 37,5 & 1,00 & 1,00 \\
\hline Renda familiar (salários mínimos) & & $p<0,001 * *$ & $p=0,004$ ** \\
\hline $0,0-0,9$ & 68,2 & $1,48(1,31-1,68)$ & $1,16(1,02-1,32)$ \\
\hline $1,0-1,9$ & 64,8 & $1,41(1,26-1,58)$ & $1,13(1,00-1,27)$ \\
\hline $2,0-3,9$ & 52,2 & $1,13(1,00-1,28)$ & $0,99(0,87-1,12)$ \\
\hline 4,0 ou mais & 46,0 & 1,00 & 1,00 \\
\hline $\begin{array}{l}\text { Se a mãe exerceu atividade } \\
\text { remunerada durante a gravidez }\end{array}$ & & $p<0,001 * \star *$ & $p=0,141 * \star \star$ \\
\hline Não & 63,1 & $1,22(1,14-1,31)$ & $1,06(0,98-1,14)$ \\
\hline Sim & 51,6 & 1,00 & 1,00 \\
\hline \multicolumn{4}{|l|}{ ॥ } \\
\hline Número de moradores por domicílio & & $p<0,001 * *$ & $p<0,001$ ** \\
\hline $1-2$ & 49,8 & 1,00 & 1,00 \\
\hline $3-4$ & 58,0 & $1,16(1,07-1,27)$ & $1,16(1,07-1,26)$ \\
\hline 5 ou mais & 72,6 & $1,46(1,33-1,59)$ & $1,35(1,23-1,48)$ \\
\hline Índice de bens (quartis) & & $p<0,001$ ** & $p<0,001$ ** \\
\hline Primeiro (pior) & 72,8 & $1,54(1,39-1,69)$ & $1,25(1,11-1,41)$ \\
\hline Segundo & 59,9 & $1,26(1,14-1,41)$ & $1,09(0,97-1,22)$ \\
\hline Terceiro & 52,8 & $1,11(0,99-1,25)$ & $1,05(0,94-1,18)$ \\
\hline Quarto (melhor) & 47,3 & 1,00 & 1,00 \\
\hline \multicolumn{4}{|l|}{ III } \\
\hline Número de filhos tidos & & $p<0,001$ ** & 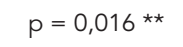 \\
\hline Nenhum & 50,7 & 1,00 & 1,00 \\
\hline $1-2$ & 59,1 & $1,16(1,07-1,26)$ & $1,51(0,95-2,38)$ \\
\hline 3 ou mais & 72,6 & $1,43(1,32-1,55)$ & $1,67(1,05-2,66)$ \\
\hline
\end{tabular}

(continua) 
Tabela 3 (continuação)

\begin{tabular}{|c|c|c|c|}
\hline \multirow[t]{2}{*}{ Nível/Variável } & \multirow{2}{*}{$\begin{array}{c}\text { Prevalência de } \\
\text { inadequação (\%) }\end{array}$} & \multicolumn{2}{|c|}{ RP (IC95\%) } \\
\hline & & Bruta & Ajustada \\
\hline \multicolumn{4}{|l|}{ III } \\
\hline Se teve algum aborto & & 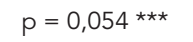 & $p=0,548 * \star \star$ \\
\hline Não & 64,7 & 1,00 & 1,00 \\
\hline Sim & 58,6 & $0,90(0,82-1,00)$ & $0,97(0,88-1,07)$ \\
\hline Se planejou a gravidez & & $p<0,001 * \star \star$ & $p=0,225 * \star \star$ \\
\hline $\operatorname{Sim}$ & 49,6 & 1,00 & 1,00 \\
\hline Mais ou menos & 53,9 & $1,09(0,94-1,25)$ & $0,97(0,81-1,16)$ \\
\hline Não & 64,4 & $1,30(1,20-1,40)$ & $1,07(0,97-1,18)$ \\
\hline \multicolumn{4}{|l|}{ IV } \\
\hline Tipo de médico que fez pré-natal & & $p<0,001 \star \star \star$ & $\mathrm{p}=0,002 \star \star \star$ \\
\hline Convênio/Particular & 47,7 & 1,00 & 1,00 \\
\hline ESF & 52,2 & $1,09(0,98-1,22)$ & $0,86(0,74-0,99)$ \\
\hline Tradicional & 72,1 & $1,51(1,37-1,66)$ & $1,07(0,94-1,23)$ \\
\hline Ambulatório & 65,1 & $1,36(1,25-1,49)$ & $1,08(0,95-1,22)$ \\
\hline Intercorrências durante a gestação \# & & $p<0,001 * \star$ & $p<0,001 * \star$ \\
\hline Nenhuma & 67,2 & $1,35(1,21-1,50)$ & $1,38(1,20-1,59)$ \\
\hline $1-2$ & 57,0 & $1,14(1,03-1,27)$ & $1,23(1,08-1,40)$ \\
\hline 3 ou mais & 49,9 & 1,00 & 1,00 \\
\hline Total & 58,2 & & \\
\hline
\end{tabular}

ESF: Estratégia Saúde da Família; IC95\%: intervalo de 95\% de confiança; RP: razão de prevalência.

* Pré-natal inadequado segundo Silveira et al.: início do pré-natal a partir do quinto mês e/ou realização de cinco consultas ou menos e/ou realização de somente um ou nenhum dos seguintes exames laboratoriais: hemograma, exame de urina e sífilis (VDRL);

** Teste de tendência linear;

$\star \star \star$ Teste de heterogeneidade;

\# Doença hipertensiva específica da gestação, hipertensão arterial sistêmica, diabetes mellitus, depressão, anemia, ameaça de aborto, trabalho de parto prematuro, corrimento vaginal, infecção do trato urinário, sangramento vaginal nos últimos três meses de gestação.

Os critérios aqui mencionados foram amplamente utilizados em estudos conduzidos em Pelotas 7 e Rio Grande 9 (Estado do Rio Grande do Sul); Ribeirão Preto (São Paulo); São Luís (Maranhão) 14, e em municípios menores nas regiões Norte e Nordeste do Brasil 2. Nem mesmo o PHPN, versão oficial do governo federal, tem sido utilizado para avaliar a adequação do pré-natal, exceto pelo próprio Ministério da Saúde.

Segundo o critério utilizado por Takeda, o índice de inadequação do pré-natal para Rio Grande em 2010 foi de 28\%. Em Pelotas, em 1998, esse índice foi de $63 \%$ 7, e em Caracol e Anísio de Abreu (Piauí), em 2008, foi de 19\% 2. Utilizandose Coimbra et al., o índice de inadequação para
Rio Grande foi, em 2010, de 27\%, e em São Luís, em 1997/1998, foi de 53\%, e em Ribeirão Preto, 43\% em 2004 14. Finalmente, para Silveira et al., o índice de inadequação para Rio Grande foi de 58\%, contra 69\% em Pelotas em 19987 e $82 \%$ em Rio Grande em 20079.

A comparação desses valores deve ser feita com muito cuidado porque, além da diferença entre as medidas serem de até 13 anos, houve estudo que trabalhou com dado secundário 2 ou que não incluiu gestantes de áreas rurais 14, onde a prevalência de pré-natal inadequado tende a ser maior em virtude da maior dificuldade de acesso a este tipo de serviço. Apesar dessas limitações, é possível constatar, no caso do Município de Rio Grande, que a proporção de gestantes 
com pré-natal inadequado, segundo critério utilizado por Silveira et al., diminuiu cerca de $40 \%$ em três anos, caindo de $82 \%$ em $2007{ }^{9}$ para $58 \%$ em 2010. Aqui é importante salientar que na análise específica buscando avaliar a qualidade do pré-natal dentro dos serviços públicos de saúde no município, observou-se maior índice de inadequação entre mães que realizaram a maioria das consultas nas UBS tradicionais e em ambulatórios, em comparação àquelas atendidas em UBS com Estratégia Saúde da Família 15. Embora a qualidade do pré-natal nesse município venha melhorando, deve-se destacar que, por esse tipo de serviço não requerer recursos tecnológicos de alta complexidade e de poder ser ofertado não somente pelo médico, mas, quando de baixo risco, também pela enfermeira, esses índices ainda são muito elevados.

Das 13 variáveis incluídas no modelo hierárquico de análise, que foram as mesmas para os três desfechos avaliados, todas se mostraram associadas ao seu respectivo desfecho para um valor de p de até 0,20. Após ajuste, 11 delas mantiveram-se significativamente associadas ao critério utilizado por Takeda e Coimbra et al. (Tabela 4), mas apenas oito em relação a Silveira et al. As mesmas sete variáveis estiveram associadas $(p \leq 0,05)$ aos três desfechos, mas, ao se considerar apenas Takeda e Coimbra et al., este número subiu para 10. Isso sugere que esses dois índices têm praticamente os mesmos determinantes. No caso de Silveira et al., isso modifica um pouco. Por incluir alguns exames laboratoriais, esse índice é claramente mais exigente e, portanto, mais robusto porque sofre influência de um número menor de variáveis, o que o torna mais dificilmente modificável e, em consequência, mais propício à avaliação da assistência pré-natal.

As razões de prevalência (RP) mostram uma vez mais a disparidade no acesso a serviços de pré-natal entre as riograndinas. Considerando os três critérios avaliados, verifica-se que a RP de inadequação para mães que não vivem com companheiro, que possuem baixa escolaridade $\mathrm{e}$ renda familiar, com maior número de moradores e menor disponibilidade de bens no domicílio é substancialmente maior em relação às gestantes melhor classificadas em cada uma destas variáveis. Estudos conduzidos em Pelotas, e em diversas outras localidades brasileiras mostram que a oferta de serviço melhora a cobertura, mas ao não considerar eventuais iniquidades de acesso, torna-se perverso porque aumenta a disparidade entre ricos e pobres, compromete o custo-efetividade das intervenções e, por conseguinte, tem baixo impacto sobre os índices de morbimortalidade materno-infantil 16.

O uso de critérios para avaliação da adequação da assistência pré-natal é por demais necessário. Não parece plausível que a simples medida do número de consultas de pré-natal, bem como da idade gestacional em que esta consulta é realizada possa, de fato, significar que

Tabela 4

Variáveis incluídas no modelo hierárquico de análise que se mostraram significativamente associadas ao seu respectivo desfecho após ajuste.

\begin{tabular}{|c|c|c|c|}
\hline \multirow[t]{2}{*}{ Variável } & \multicolumn{3}{|c|}{ Critério } \\
\hline & Takeda 5 & Coimbra et al. 8 & Silveira et al. 7 \\
\hline 1. Idade da mãe & $\mathrm{S}$ & $\mathrm{S}$ & $\mathrm{N}$ \\
\hline 2. Cor da pele & $\mathrm{S}$ & $\mathrm{S}$ & $\mathrm{N}$ \\
\hline 3. Vive com o companheiro & $\mathrm{S}$ & $\mathrm{S}$ & $\mathrm{S}$ \\
\hline 4. Escolaridade & $\mathrm{S}$ & $\mathrm{S}$ & $\mathrm{S}$ \\
\hline 5. Renda familiar & $\mathrm{S}$ & $\mathrm{S}$ & $\mathrm{S}$ \\
\hline 6. Se a mãe exerceu atividade remunerada durante a gravidez & $\mathrm{S}$ & $\mathrm{N}$ & $\mathrm{N}$ \\
\hline 7. Número de moradores por domicílio & $\mathrm{S}$ & $\mathrm{S}$ & $\mathrm{S}$ \\
\hline 8. Índice de bens em quartis & $\mathrm{S}$ & $\mathrm{S}$ & $\mathrm{S}$ \\
\hline 9. Número de filhos tidos & $\mathrm{S}$ & $\mathrm{S}$ & $\mathrm{S}$ \\
\hline 10. Se teve algum aborto & $\mathrm{N}$ & $\mathrm{N}$ & $\mathrm{N}$ \\
\hline 11. Se planejou a gravidez & $\mathrm{S}$ & $\mathrm{S}$ & $\mathrm{N}$ \\
\hline 12. Se fez pré-natal no serviço público ou privado & $\mathrm{S}$ & $\mathrm{S}$ & $\mathrm{S}$ \\
\hline 13. Intercorrências durante a gestação & $\mathrm{N}$ & $\mathrm{S}$ & $\mathrm{S}$ \\
\hline
\end{tabular}

N: não; S: sim. 
os cuidados mínimos a um pré-natal adequado estejam garantidos. Há, portanto, necessidade da utilização de critérios mais robustos como o de Silveira et al., por exemplo. Mas há também que ressaltar que desde que esse critério foi proposto, há mais de uma década, novos cuidados passaram a ser obrigatórios no pré-natal como, por exemplo, a realização do anti-HIV, sorologia para hepatite B e toxoplasmose 17. Além disso, é preciso rever se os aspectos considerados no critério utilizado por Silveira et al. continuam tendo a mesma importância clínica e relevância epidemiológica.

\section{Resumen}

Este estudio midió la prevalencia e identificó los factores asociados a la inadecuación del cuidado prenatal, conforme diferentes criterios entre puérperas residentes en municipio de Río Grande, Río Grande do Sul, Brasil, en 2010. Se aplicó un cuestionario patrón, hasta 24 horas después del parto, a todas las madres que tuvieran un hijo en cualquiera de las dos maternidades locales. Se utilizó el test de chi-cuadrado para comparar proporciones y la regresión de Poisson con ajuste robusto de la variancia en el análisis multivariable. Se entrevistaron a 2.395 madres $(97,2 \%$ del total). Los índices de inadecuación del cuidado prenatal fueron $28 \%, 27 \%$ y 58\%, según Takeda, Coimbra et al. y Silveira et al., respectivamente. La razón de prevalencias, respecto a la realización de cuidado prenatal inadecuado, fue mayor entre las madres pobres. Tras el ajuste, casi todas las variables del modelo se mostraron significativamente asociadas a la inadecuación del cuidado prenatal para Takeda y Coimbra et al., en contraste con Silveira et al. Se observó la necesidad de mejorar la calidad del cuidado prenatal y que el índice propuesto por Silveira et al. es más robusto.

Atención Prenatal; Evaluación de Proceso (Atención de Salud); Calidad de la Atención de Salud
É notório o progresso na assistência à gestação e ao parto Brasil afora. Assim como é evidente o avanço científico na busca de critérios que meçam de forma mais completa a qualidade dos serviços oferecidos a essas populações. Persiste, no entanto, a necessidade de criar algum critério que avalie também a qualidade do exame clínico oferecido durante as consultas de pré-natal. As propostas de avaliação existentes resumem-se à contagem de eventos recebidos ao longo do período gestacional, o que é claramente insuficiente no que diz respeito à determinação da qualidade do serviço oferecido.

\section{Colaboradores}

J. S. Saavedra foi responsável pela revisão bibliográfica, análise de dados e redação do artigo. J. A. Cesar delineou o estudo, preparou o questionário, supervisionou o trabalho de campo, a entrada e auxiliou na análise de dados e redação do artigo.

\section{Agradecimentos}

Aos comentários do Prof. Dr. Juvenal S. Dias-da-Costa e do Prof. Dr. Raúl Mendoza-Sassi que muito contribuíram para elaboração deste artigo. 


\section{Referências}

1. Gonçalves CV, Cesar JA, Mendoza-Sassi RA. Qualidade e eqüidade na assistência à gestante: um estudo de base populacional no Sul do Brasil. Cad Saúde Pública 2009; 25:2507-16.

2. Dias-da-Costa JS, Cesar JA, Haag CB, Watte G, Vicenzi K, Schaefer R. Inadequacy of prenatal care in underprivileged parts of the Northeast of Brazil: prevalence and some associated factors. Rev Bras Saúde Matern Infant 2013; 13:101-9.

3. Chrestani MA, Santos IS, Cesar JA, Winckler LS, Gonçalves TS, Neumann NA. Assistência à gestação e ao parto: resultados de dois estudos transversais em áreas pobres das regiões Norte e Nordeste do Brasil. Cad Saúde Pública 2008; 24:1609-18.

4. Kotelchuck M. An evaluation of the Kessner Adequacy of Prenatal Care Index and a proposed Adequacy of Prenatal Care Utilization Index. Am J Public Health 1994; 84:1414-20.

5. Takeda S. Avaliação da qualidade do pré-natal: programa para gestantes em unidade de atenção à saúde [Dissertação de Mestrado]. Pelotas: Universidade Federal de Pelotas; 1993.

6. Área Técnica de Saúde da Mulher, Departamento de Ações Programáticas Estratégicas, Secretaria de Atenção à Saúde, Ministério da Saúde. Prénatal e puerpério: atenção qualificada e humanizada - manual técnico. Brasília: Ministério da Saúde; 2006.

7. Silveira DS, Santos IS, Costa JS. Atenção pré-natal na rede básica: uma avaliação da estrutura e do processo. Cad Saúde Pública 2001; 17:131-9.

8. Coimbra LC, Silva AA, Mochel EG, Alves MT, Ribeiro VS, Aragão VM, Bettiol H. Factors associated with inadequacy of prenatal care utilization. Rev Saúde Pública 2003; 37:456-62.

9. Cesar JA, Mano OS, Carlotto K, Gonzalez-Chica DA, Mendoza-Sassi RA. Público versus privado: avaliando a assistência à gestação e ao parto no extremo sul do Brasil. Rev Bras Saúde Matern Infant $2011 ; 11: 257-63$.
10. Programa das Nações Unidas para o Desenvolvimento. Desenvolvimento humano e IDH. http:// www.pnud.org.br/atlas/ranking/Ranking-IDHMMunicipios-2010.aspx (acessado em 05/Fev/2014).

11. Barros AJD, Hirakata UM. Alternatives for logistic regression in cross-sectional studies: an empirical comparision of models that directly estimate the prevalence ratio. BMC Med Res Method 2003; 3:21.

12. Victora CG, Huttly SR, Fuchs SC, Olinto MTA. The role of conceptual frameworks in epidemiological analysis: a hierarchical approach. Int J Epidemiol 1997; 26:224-47.

13. Kirkwood R, Sterne JAC. Essentials of medical statistics. 2nd Ed. London: Blackwell Science Ltd. 2003.

14. Coimbra LC, Figueiredo FP, Silva AAM, Barbieri MA, Bettiol H, Caldas AJM, et al. Inadequate utilization of prenatal care in two Brazilian birth cohorts. Braz J Med Biol Res 2007; 40:1195-202.

15. Cesar JA, Sutil AT, Santos GB, Cunha CF, MendozaSassi RA. Assistência pré-natal nos serviços públicos e privados: estudo transversal de base populacional em Rio Grande, Rio Grande do Sul, Brasil. Cad Saúde Pública 2012; 28:2106-14.

16. Victora CG, Cesar JA. Saúde materno-infantil no Brasil: padrões de morbidade e possíveis intervenções. In: Rouquayrol MZ, Almeida-Filho NM, organizadores. Epidemiologia e saúde. 6a $\mathrm{Ed}$. Rio de Janeiro: Medsi; 2003. p. 415-67.

17. Departamento de Atenção Básica, Secretaria Atenção à Saúde, Ministério da Saúde. Atenção ao prénatal de baixo risco. Brasília: Ministério da Saúde; 2012.

Recebido em 04/Jun/2014

Versão final reapresentada em 13/Nov/2014

Aprovado em 08/Dez/2014 